\title{
Collaborative Learning in an Online-only Design for Manufacturability Course
}

\section{Miss Taylor Tucker, University of Illinois at Urbana - Champaign}

Taylor Tucker graduated from the University of Illinois at Urbana-Champaign with a Bachelor's degree in engineering mechanics. She is interested in engineering design and lends her technical background to her research with the Collaborative Learning Lab, exploring how to improve ill-structured tasks for engineering students in order to promote collaborative problem solving and provide experience relevant to authentic work in industry. She also writes for the Department of Mechanical Science and Engineering at Illinois and is active on the leadership \& administration team for the University's Women in Engineering chapter.

\section{Mr. Nattasit Dancholvichit, University of Illinois at Urbana - Champaign}

Nattasit Dancholvichit was born in Bangkok, Thailand in 1990. He received a B.A. degree in mechanical engineering from the University of Michigan in 2014. He received an M.S. degree in mechanical engineering from the University of Illinois Urbana-Champaign in 2017. He is currently a Ph.D. student in Mechanical Science and Engineering at the University of Illinois Urbana Champaign. He carries out research in the field of micro-manufacturing, precision control, manufacturing, and mechatronics. His research also includes control optimization and system identification. He is also a graduate teaching assistant of design for manufacturability.

\section{Dr. Leon Liebenberg, University of Illinois at Urbana - Champaign}

Leon is a Teaching Associate Professor in mechanical science and engineering at the University of Illinois at Urbana-Champaign. He is also a Fellow of the UIUC's Center for Innovation in Teaching and Learning. Before coming to UIUC, he was a professor of mechanical engineering at two South African universities (University of Pretoria; North West University) and a higher education consultant in Switzerland where he worked with colleges of engineering and technology management. Leon is passionate about multidisciplinary research, particularly in the fields of energy engineering, biomedical engineering, and engineering education. Together with UIUC colleagues in the ENGagement In eNgineering Education (ENGINE) instructional innovation team, Leon is evaluating a wide array of pedagogies of engagement. The intention is to promote deep learning and improved engagement of students in subject matter. Leon teaches a variety of subjects, including: Innovation; Statics; Dynamics; Thermodynamics; Fluid Dynamics; Design for Manufacturability; Mechanical Design; Heat Transfer; Energy Conversion Systems; Aerodynamics; Aeronautics; and Advanced Heat and Mass Transfer. He holds a doctoral and two master's degrees from Imperial College London and from the University of Johannesburg. 


\title{
WIP: \\ Collaborative Learning in an Online-Only Design for Manufacturability Course
}

\begin{abstract}
In contemporary design-for-manufacturability education, the use of design-thinking (or humancentered design) and team-based design projects are ubiquitous. Students are typically taken on a journey to better appreciate synthesis of the "big picture" while learning to consider an openended manufacturability problem from various perspectives and discovering the value in empathy and co-creation. However, with the onset of online-only modes of instruction to cope with the Covid-19 pandemic, meaningful collaborative learning has become challenging. Students regularly cite the lack of social interaction as a main reason for poor virtual teamwork and tend to display entrenched preference for face-to-face interactions to perform ideation and to understand or resolve issues, which hampers the use of online counterparts.

However, online learning has brought to light several digital platforms that are easily customizable for online collaboration among students. When it comes to virtual ideation (or virtual "brainstorming"), effective generation of new ideas or concepts is difficult. Social media platforms like WeChat, GroupMe, and Discord are beneficial for high-level idea sharing; Zoom and other videoconferencing platforms might be similarly helpful. Still, engineering students tend to have trouble when using computer-aided drawing platforms that do not allow interactive collaboration in real-time (e.g. Fusion 360). Instructors also must contend with student apprehension to use unfamiliar digital tools. Among online collaborative platforms, Miro may pose a solution to these challenges, as it allows for synchronous interaction and captures essential elements of a face-to-face ideation environment. This platform could also facilitate empathy-mapping and journey-mapping labs, where team members would capture the team's combined user knowledge and map-out user attitudes, behaviors, needs, and pain points.

This study investigates if and how the use of the Miro virtual ideation platform affects ideation of small teams of engineering students $(n=65)$ in a sophomore-level design-formanufacturability course. Questionnaires were used to evaluate 1) students' perceived cognitive and emotional engagement when using Miro, 2) Miro's utility in authentically subjecting students to aspects of design-thinking, and 3) the degree of psychological safety in Miro's virtual collaborative environment. The effective use of online ideation tools, like Miro, is of paramount importance when engineering students are collaborating in an online-only learning environment. Findings from this study will provide insight toward the utility in adopting Miro (or similar platforms) for such purposes as well as help identify psychological issues that could be suitably addressed when using online collaborative platforms such as Miro. This study contributes to the body of knowledge pertaining to effective student engagement during online or hybrid modes of education.
\end{abstract}




\section{Background}

The modern engineering industry requires enhanced capacity for self-direction, collaboration, cooperation, accountability, innovation, and adaptation to change [1]. These skills are especially necessary for facilitating successful human-centered design. In contemporary engineering design education, the use of design-thinking (or human-centered design) and team-based design projects are ubiquitous [2], [3]. Engineering students are taken on a journey to better appreciate synthesis of the "big picture" whilst learning to consider an open-ended problem from various perspectives. Along the way, they also discover the values of empathizing, both with teammates and clients/end-users, and co-creating solutions. They are routinely required to engage in collaborative learning activities such as team projects, team presentations, group discussions, and peer evaluations, all of which require significant collaboration [4].

With the onset of online-only modes of instruction to cope with the Covid-19 pandemic, meaningful collaborative learning has become challenging [5]. Students regularly cite the lack of social interaction as a main reason for poor virtual teamwork; indeed, interaction among team members (i.e. the social state of the team) is a significant factor for effective team-based project work and may be more challenging to sustain in a virtual learning environment [6], [7]. Furthermore, students display entrenched preferences for face-to-face interactions to perform ideation and to understand or resolve issues, which hampers the use of online counterparts [8]. Even selecting a viable candidate for virtual ideation (or virtual "brainstorming") is difficult, as the generation of new concepts is inherently challenging under face-to-face (i.e. "normal") circumstances. Social media platforms like WeChat, GroupMe, and Discord may be beneficial for high-level idea-sharing; Zoom and other videoconferencing platforms might be similarly helpful. Although these platforms tend to lack the structure necessary for continuous feedback, some can provide instant media sharing to help visualize the workflow. Despite these resources, engineering students have faced collaborative hurdles, such as experiencing difficulty using computer-aided drawing platforms that lack Cloud collaboration, needing to address social loafing, and navigating synchronous and asynchronous collaboration, especially across multiple time zones [9].

However, online learning has also brought increased attention to digital platforms that are easily customizable for virtual collaboration among students [10]. Among these, Miro [11] is a popular one that allows for real-time interaction. Miro features several resources that can be beneficial for supporting virtual collaboration: idea boards (or whiteboards) that come with a large selection of templates (diagrams and flowcharts); colorful "sticky notes" that allow collaborators to emulate face-to-face low-fidelity design synthesis processes; a real-time display that indicates active team members; empathy-mapping templates to assist teams in mapping end users' attitudes, behaviors, needs and pain points; and journey-mapping templates to assist teams in capturing their design process. Unlike many of its competitors, Miro supports both discrete and continuous brainstorming processes, meaning that users can visualize either independent steps and thoughts throughout the process or a continuous flow. These features allow the platform to convey "rich media" that suits the task according to media richness theory [12]. Miro also offers several functions, such as cursor position of each user on the screen, activity logs, and personalized notes, to uplift social presence - a vital element in online classroom settings. These 
features define Miro as a strong tool for online or hybrid learning environments that require high levels of collaboration [12].

In this study, we use mixed methods to investigate if and how the use of the Miro platform affects engineering students' ideation in small groups during virtual laboratory sections of a sophomore-level Design for Manufacturability course ( $n=61$ participants). We conduct a comprehensive analysis of students' use of Miro's virtual collaboration platform and evaluate students' perceptions regarding the utility of Miro for performing virtual ideation in collaborative projects are evaluated. This study also explores connections between students' cognitive and emotional engagement while using Miro. Secondary objectives include 1) evaluating students' mastery of (or competence in) subject- and related design-thinking contents when using Miro and 2) interrogating students' perceived degree of psychological safety in a virtual collaborative environment (in terms of communication, cooperation, engagement, and permitted failure/experimentation). This study seeks to answer the following research question: How did the use of Miro impact groups' virtual collaboration and students' perception of collaboration?

\section{Pedagogical Approach}

Students in this online-only course were subjected to two 2-hour synchronous lab sessions (via Zoom) in the first two weeks of class. Students were divided into sections of 10-15 students, then subdivided into 2-3 teams of 5 students each. Each lab session was facilitated by two graduate teaching assistants. Students were presented with a design challenge ("redesign an existing product at your homes, e.g. a ballpoint pen") and tasked with having to use the Miro virtual collaborative platform to facilitate their design process. During certain stages of the lab session, teams shared their design ideas with one another.

\section{Lab session 1}

In the first week's lab session, teams were reminded of the salient features of design thinking and the importance of need finding, empathizing, and co-creation. Teams then had to find simple products at their homes/dormitories that could be redesigned to address a need identified by the team (e.g. ergonomically redesigning a ballpoint pen for a handicapped person to use). Team members were prompted to first share their perceptions of the current ease of use of the product and then disassemble it and create an annotated parts list on Miro. Team members then shared for whom (which stakeholder) the product would be redesigned; subsequent research and roleplay was used to unanimously select a final design candidate. Team members then used Miro's extensive graphics capability to draw a mind map of their proposed redesigns, citing stakeholders, mechanical components, and anticipated manufacturing and recycling processes. Teams also generated and posted a "point of view" statement that served to guide each member's sketches of how the product should be redesigned. The teams' collaborative design works were then saved for review in the next lab.

Lab session 2

In the second week of their lab sessions, teams provided peer feedback on proposed ideas and then used their own feedback to select a finalized concept. Teams then modified their Miro product mind maps and performed online searches for typical part and product costs, which were listed in their final annotated design. Like conventional laboratory reports, teams generated 
Miro-based reports that contained all their work pertaining to the design-thinking sequence of activities (i.e. empathizing, defining, ideating, prototyping, and testing). Each team then presented their designs to their lab section and had the opportunity to vote for a best design, which earned extra credits for the winning team members. Figure 1 shows an extract of one winning team's Miro board.

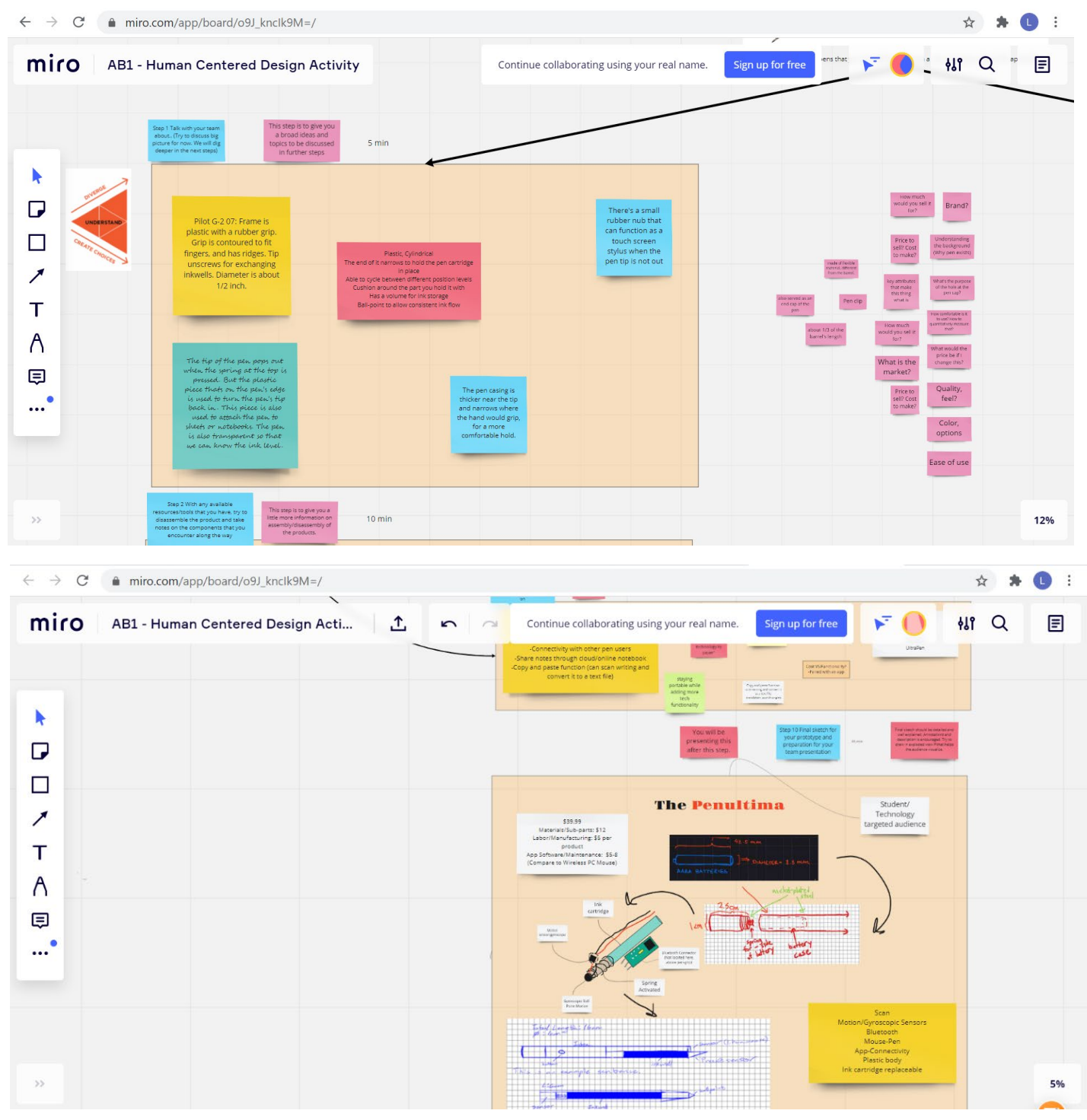

Figure 1. Extracts of a Miro idea board used by a mini-project team in ME 270 (Design for Manufacturability). https://miro.com/app/board/o9J knclk9M=/ (Registration on the Miro platform is required to gain access.)

\section{Methodology}

\section{Design}

This study is part of an ongoing exploration of pedagogies of engagement that began with investigating the use of ePortfolios in a sophomore-level Design for Manufacturability course. Qualitative questionnaires were used to investigate students' engagement with course content 
using the virtual collaboration platform Miro. The questionnaire content had three thrusts: to evaluate students' perceived cognitive and emotional engagement when using Miro; to explore the utility of Miro by authentically subjecting students to aspects of design-thinking, and to investigate the degree of psychological safety in Miro virtual collaborative environment (in terms of communication, cooperation, engagement, permitted failure/experimentation). Appendix 1 lists the questionnaire questions. Questionnaires were delivered online to individual participants; participant identifiers were removed prior to analysis.

\section{Participants}

Participants were 61 undergraduate students in a Fall 2020 sophomore-level Design for Manufacturability course (13 female, 47 male, 1 other). All students participated in the course activities, but only research participants provided feedback via an online survey that was approved by the University's IRB. Based on previous participation levels [13], a larger sample size was expected for this study at the onset. Unforeseen factors related to the Covid-19 pandemic likely impacted students' ability and willingness to participate.

\section{Analysis}

Student engagement was measured using a series of questions to evaluate cognitive engagement and emotional engagement that were devised by following the guidelines and factorgroupings in Halverson and Graham's extensive meta-study [14]. All questions were written in such a manner that aligns positive values with a desired agreement response. Response options corresponded to the following Likert scale: strongly disagree, disagree, slightly disagree, slightly agree, agree, strongly agree. Questions were preassigned to the following eight factors: conceptual understanding, creativity, grasp of design skills, ease of asynchronous collaboration, willingness to embrace risk, organization, perceived roles of sketching, and teamwork. All responses were coded numerically to indicate positive and negative tendencies; "strongly disagree" corresponded to -3 , "disagree" to -2 , "strongly agree" to 3 , and so on. Factor analysis was used to correlate students' perceived emotional and cognitive engagement to task-related experiences.

\section{Results}

\section{Quantitative}

Questionnaire prompts were grouped according to the above factors. Figure 2 summarizes the results captured by these factors. A tendency toward the positive $\mathrm{x}$-axis indicates a more positive response to the factor captured by the questionnaire prompt; in other words, a higher positive value means that students on average tended to more strongly agree/identify with the prompt. A negative value indicates that students on average tended to disagree or did not identify with the prompt.

The data reveal that students tended to have positive experiences while using Miro for online collaboration. Table 1 presents statistical data of the 20 questions. 


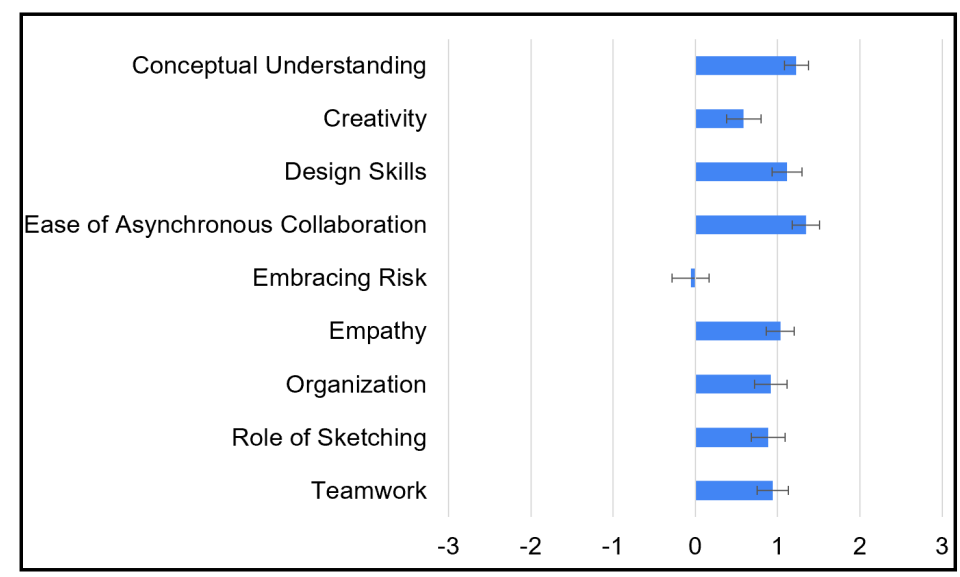

Figure 2. Questionnaire results of all participants. Out of the class population of 118 students, 64 participated.

Table 1. Descriptive Statistics by Factor

\begin{tabular}{|c|c|c|c|}
\hline Factor & Mean, $\overline{\boldsymbol{X}}$ & Standard Deviation & Standard Error \\
\hline $\begin{array}{c}\text { Conceptual } \\
\text { Understanding }\end{array}$ & 1.230 & 1.494 & 0.191 \\
\hline Creativity & 0.590 & 1.590 & 0.204 \\
\hline Design Skills & 1.115 & 1.561 & 0.200 \\
\hline $\begin{array}{c}\text { Ease of Asynchronous } \\
\text { Collaboration }\end{array}$ & 1.344 & 1.342 & 0.172 \\
\hline Embracing Risk & -0.057 & 1.771 & 0.227 \\
\hline Empathy & 1.033 & 1.292 & 0.165 \\
\hline Organization & 0.918 & 1.411 & 0.181 \\
\hline Role of Sketching & 0.885 & 1.643 & 0.210 \\
\hline Teamwork & 0.940 & 1.165 & 0.149 \\
\hline
\end{tabular}

Table 1 and Figure 2 show that participants responded positively (averaging responses of at least "Slightly Agree" on a six-point Likert scale ranging from "Strongly Disagree" to "Strongly Agree," $\bar{X} \geq 1.000$ ) for factors pertaining to conceptual understanding, grasp of design skills, ease of asynchronous collaboration, and empathy. The use of Miro was deemed to be of lesser significance $(-0.300<\bar{X}>0.300)$ for factors pertaining to embracing risk. The use of Miro received mixed perception on average for factors pertaining to creativity and the role of sketching, while factors pertaining to organization and teamwork tended to receive positive responses. These results are also reflected in students' qualitative responses.

Figures 3 and 4 present data of male-only and female-only students, respectively. For these groups, the participant who identified as "other" has been excluded. Figures 3 and 4 reveal a gender disparity for factors pertaining to willingness to embrace risk, with female students displaying less willingness on average than male students. The female participant group also has 
noticeably larger margins of error than the male group, indicating a wider range of responses among female participants. The two groups display the most positive response for factors pertaining to ease of asynchronous collaboration.

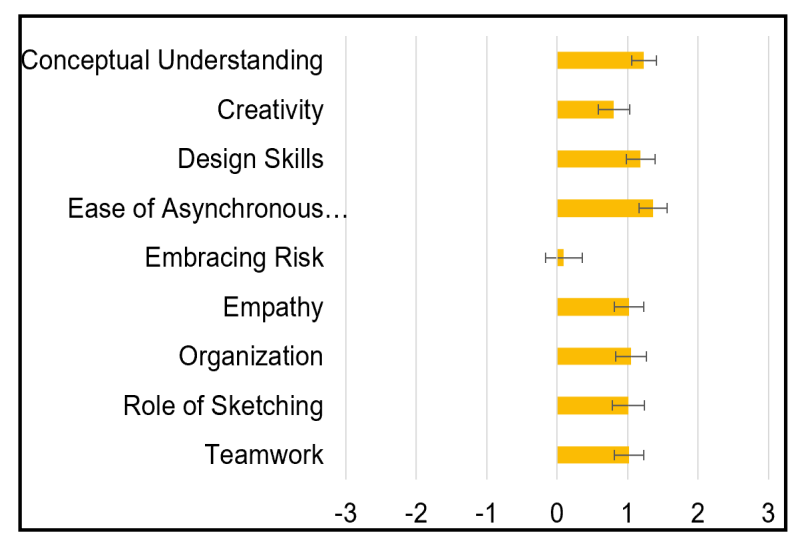

Figure 3. Questionnaire results for all participants who identified as male $(n=47)$.

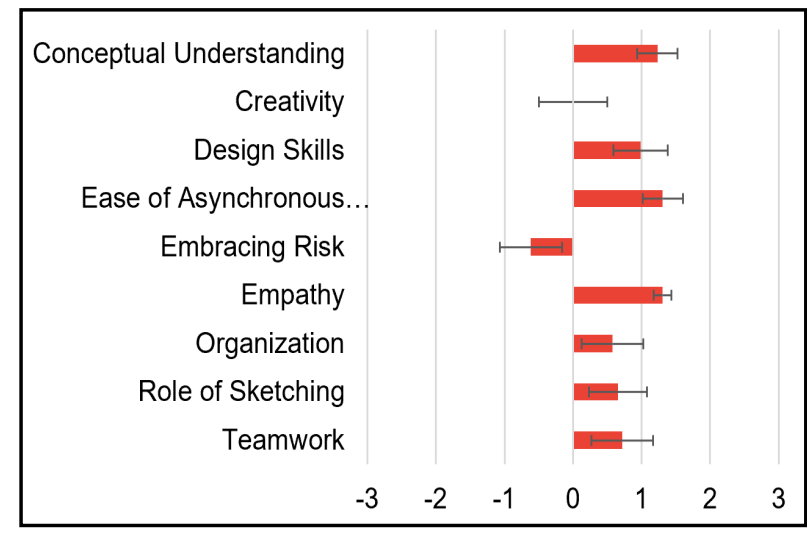

Figure 4. Questionnaire results for all participants who identified as female $(n=13)$.

Qualitative

The questionnaires also asked students to reflect on how much they might have benefited from using Miro for idea generation and presentation of their design ideas. Below is a representation of salient comments.

\section{Positive Perceptions}

Miro was helpful in sharing ideas in a way that we can easily visualize offline. Elaborating on those ideas were also easy. But creativity was not necessarily facilitated by Miro solely. It depended on each team member's effort.

I really liked Miro because it makes everything super easy to follow, and it's colorful and engaging, so it's a win-win. I really liked in the lab when we designed a modified object (pen) and we went through the design process and made designs. I'm someone who loves to draw and the whole designing process of a product so I thought that was super fun. I think 
Miro is a great platform for sharing design ideas. Compared to using Zoom, the teammates can better visualize different design ideas.

It was excellent for brainstorming and laying out large scale ideas.

I very much enjoyed the visual aspect of Miro. It was a largely intuitive user interface and really helped all of our team members to communicate their design ideas more effectively because of the great propensity to share graphics and sketches through Miro.

I really enjoyed using Miro. It was a new platform for me, and I really liked how multiple users could view and edit at the same time. I liked how you had multiple options to edit through text and sketch. It was just nice to show your work without having to have one individual share their screen at a time. The ability to visually compare design concepts side by side and show design progression without having to navigate through messages saved time and fostered clearer communication.

Negative and Neutral Perceptions

My teammates left most of the Miro work to me. In Zoom, you can tell when someone hasn't talked for a few minutes. Using Miro, students feel comfortable doing nothing. The only one who noticed was myself, because I wrote most.

Miro was helpful for planning and brainstorming ideas but using it for designing wasn't as effective as say a Zoom call. It was very beneficial to get a starting concept, but after that wasn't the most useful.

One complaint I would have is that importing and formatting those sketches and graphics in Miro was quite difficult and frustrating at times.

Ultimately, we were using Miro to present a cleaned-up version of our analysis. Most of the backend work took place over Google Docs or Zoom, as we found those platforms easier to use.

Some of the editing and other functions were annoying sometimes. Although a solid website in theory, my group found that we enjoyed using Zoom, Snapchat, and shared Google Docs more than Miro.

I think Miro is a good complement to these communicating platforms because it has the design element, but talking at the same time would be nice too.

My team did not use Miro in the projects and did not like it as a platform. We used Google Docs and GroupMe for the projects.

Miro was really helpful to format flowcharts for our projects, though I wish there was a better way to integrate the flowchart into a document or ePortfolio because the text would end up too small in screenshots. We also used Miro synchronously every time we used it and used other platforms like GroupMe to communicate asynchronously, so I can't comment much on asynchronous use of Miro, though from how I experienced it, Miro might not be best used asynchronously. 
We also asked students to make suggestions for improvement when using Miro or similar collaborative online platforms. Below is a summary of salient suggestions.

It does provide a collaborative environment, but sometimes it is just a bit hard to use or download your work. It should also be made available for free to students as it is important that we can download good quality picture or files from Miro.

Miro is too clunky for the desired application; sketching was difficult and time consuming, and importing was too. I would suggest using Zoom on a phone and propping it up to view a piece of paper and draw on that to communicate design ideas.

Miro is a great tool for a "virtual whiteboard" and I can see no other alternative. However, I would not say it can be effective for continuous team communication. That should be left for things that emphasize on social interaction (Zoom, Discord, GroupMe, Slack, etc.)

In terms of communication, Miro alone is not a great standalone service. However, when paired with Zoom, or a text chat service as a virtual whiteboard, it is helpful for visualization and collaboration.

\section{Discussion}

The effective use of online ideation tools, like Miro, is deemed to be of paramount importance when engineering students are collaborating in an online-only learning environment. The positive results reported in this study suggest that Miro (and similar virtual collaborative platforms) help overcome the challenges of virtual collaboration, including supporting teamwork and fostering empathy skills, whilst improving virtual communication.

\section{Quantitative}

It is now accepted that emotions cannot be separated from thinking in guiding rational behavior, memory retrieval, decision making, problem solving, and creativity, among others [15]. As it follows that positive emotions assist learning, it is heartening to see that participants perceived the promotion of empathy in their online collaborative experiences. These results are also supported by existing findings [5].

Within a team, it is noted that not all engineering students are comfortable with sketching. Students in a team usually organize and subdelegate their work according to their comfort level. Brainstorming activity on Miro does not force students to take risks, as the platform offers a wide variety of media to work with (e.g. flowchart, "post-it" notes, internet search function). As a result, students are less likely to embrace risk and instead stick to those tasks for which they feel capable. The asynchronous setting also provides students with opportunity for individual reflection, which allows them to contemplate and compose messages and inputs to the team. The opportunity for reflection and revision in real time may lessen the feeling of risk that they take in interacting with teammates. If so, this phenomenon should be applicable across all virtual platforms that allow for asynchronous collaboration [16].

The large margins of error shown in the male- and female-specific data may be an indication of the polarizing nature of the data, as each group reported individual choices. Both 
acceptance of Miro as a beneficial platform and disregard for Miro were reported. Overall, the quantitative results suggest that the use of the Miro online collaborative platform as part of a series of lab-based assignments increased participants' cognitive engagement (i.e. conceptual understanding, asynchronous collaboration) emotional engagement (i.e. empathy, teamwork), design skills, and general organizational skills.

\section{Qualitative}

According to feedback from students, Miro tends to be beneficial when used as a tool supplementary to synchronous video or audio conferencing (e.g. Zoom, Discord) to enhance communication within the team. It offers a wide variety of media for initiating brainstorming activity, which can help students to visualize the "big picture" of the task in early stages. However, during later stages of the task, when work is consolidated for submissions, students tend to find other familiar platforms like Google Docs to be more suitable.

From an instructor's perspective, an online collaborative platform like Miro presents a low-cost (or free), easily-accessible tool, making it attractive for contemporary learning. When this tool is properly integrated with effective pedagogies of engagement (like problem-based learning, or experiment-based learning), it can enhance students' achievement of learning goals and the development of essential skills such as teamwork, creativity, and digital skills. Also, online collaboration can facilitate peer assessment and peer learning. While it was found that Miro was an effective learning tool in an engineering laboratory-intensive course, the small sample size makes for the fact that these results cannot be generalized. A follow-up study with more participants is required.

\section{Conclusion}

Our study confirms that Miro can be easily employed to facilitate effective online collaboration, thus enhancing student engagement. This is deemed to be crucial, especially during online or hybrid modes of instruction. It is important to note that software literacy varies by student, and more conventional platforms like Google Docs tend to have a higher literacy rate. This trend may have impacted groups' perception of Miro; further integration of Miro into the curriculum should consider scaffolding for improved literacy. Furthermore, the ease of asynchronous collaboration over Miro was only measured through one question and should be more deeply investigated in future studies. Future work can also explore the adoption and perception of Miro after students have used it throughout an entire semester, especially tracking how often students choose to use it for projects that require collaboration during the brainstorming phase.

\section{Acknowledgements}

This study benefited from a grant of the Academy for Excellence in Engineering Education (AE3), University of Illinois at Urbana-Champaign. The study was approved by the UIUC IRB, protocol \#19162, "Evaluation of Pedagogies of Engagement". The authors greatly benefited from discussions with fellow team members of the ENGagement In eNgineering Education (ENGINE) strategic instructional innovations project team, University of Illinois at Urbana-Champaign: Justin Aronoff PhD, Robert Baird PhD, Cheelan Bo-Linn, Yuting Chen PhD, David Favre PhD, Tim Hale PhD, Chad Lane PhD, Kate LaBore PhD, Lewis Lehe PhD, Mina Mikhaeel PhD, 
Jessica Mingee, Shelly Schmidt PhD, Saad Shehab PhD, Esmee Vernooij, and Jim Wentworth. Thank you also to the several anonymous reviewers who provided incisive comments and excellent suggestions.

\section{APPENDIX: QUESTIONNAIRES}

Please select one answer for each of the following questions:

(strongly agree - agree - slightly agree - slightly disagree - disagree - strongly disagree)

\section{Conceptual Understanding}

Miro helped me engage with my task's content.

\section{Creativity}

Miro helped me use my creativity to effectively solve complicated problems.

\section{Design Skills}

Miro helped me evaluate my design work relative to that of my teammates.

Miro helped my team to share design ideas.

Miro helped me visualize design concepts from a "big-picture" perspective.

Miro helped my team select final design concepts.

Miro helped my team contextualize our design work.

Ease of Asynchronous Collaboration

Miro made asynchronous collaboration easier.

\section{Embracing Risk}

Miro made me more comfortable to try new approaches to solve problems.

Miro prompted me and my teammates to continually try new things.

\section{Empathy}

Miro helped me understand my teammates' perspectives.

\section{Organization}

Miro was useful for consolidating my team's research.

Miro helped my team map design work throughout our projects.

Miro helped me keep track of my teammates' contributions.

\section{Role of Sketching}

The use of drawings or sketches in Miro helped the team to better understand the problem.

With Miro, my team could import drawings or sketches easily.

Miro is useful for presenting the advantages and disadvantages of design concepts.

\section{Teamwork}

Miro helped me form a sense of belonging with my teammates.

I used Miro to actively contribute to my team's work.

Please provide some brief comments about how much you benefited from using Miro for idea generation ("ideation") and presentation of your design ideas, instead of communicating via platforms such as email, Zoom, WeChat, GroupMe, etc.: 


\section{References}

[1] A. Kamp, "Engineering Education in the Rapidly Changing World: Rethinking the Vision for Higher engineering Education," June, p. 61, 2016, [Online]. Available: http://repository.tudelft.nl/islandora/object/uuid:ae3b30e3-5380-4a07-afb5dafd30b7b433? collection=research.

[2] C. L. Dym, A. M. Agogino, O. Eris, D. D. Frey, and L. J. Leifer, "Engineering design thinking, teaching, and learning," IEEE Eng. Manag. Rev., 2006, doi:

10.1109/emr.2006.1679078.

[3] V. Taajamaa, S. Kirjavainen, L. Repokari, H. Sjoman, T. Utriainen, and T. Salakoski, "Dancing with ambiguity design thinking in interdisciplinary engineering education," in 2013 IEEE-Tsinghua International Design Management Symposium: Design-Driven Business Innovation, TIDMS 2013 - Proceedings, 2014, doi: 10.1109/TIDMS.2013.6981258.

[4] M. Hannafin, S. Land, and K. Oliver, "Open learning environments: Foundations, methods, and models," in Instructional-Design Theories and Models: A New Paradigm of Instructional Theory, 2013.

[5] A. Shahzad, R. Hassan, A. Y. Aremu, A. Hussain, and R. N. Lodhi, "Effects of COVID19 in E-learning on higher education institution students: the group comparison between male and female," Qual. Quant., 2020, doi: 10.1007/s11135-020-01028-z.

[6] Z. Mseleku, "A Literature Review of E-Learning and E-Teaching in the Era of Covid-19 Pandemic,” Int. J. Innov. Sci. Res. Technol., 2020.

[7] G. Stahl, T. Koschmann, and D. Suthers, "Cambridge Handbook of the Learning Sciences. Computer-supported collaborative learning: An historical perspective," Cambridge Handb. Learn. Sci., 2006.

[8] K. Fleischmann, "Online design education: Searching for a middle ground," Arts Humanit. High. Educ., 2020, doi: 10.1177/1474022218758231.

[9] L. P. Robert, "Behavior-Output Control Theory, Trust and Social Loafing in Virtual Teams," in Multimodal Technologies and Interaction 4.3 (2020): 39.

[10] T. A. Koszalka, Y. Pavlov, and Y. Wu, "The informed use of pre-work activities in collaborative asynchronous online discussions: The exploration of idea exchange, content focus, and deep learning," in Computers and Education, 2021, doi:

10.1016/j.compedu.2020.104067.

[11] Miro, "Miro: An Online Visual Collaboration Platform for Teamwork." www.miro.com.

[12] M. Hammond. "Online collaboration and cooperation: The recurring importance of evidence, rationale and viability," Education and Information Technologies 22.3 (2017): 1005-1024.

[13] T. Tucker, E. Vernooij, A. Wolf, Bo-C. Linn, R. Baird, N. Dancholvichit, and L. Liebenberg, "Transforming an Engineering Design Course into an Engaging Learning Experience using ePortfolios," in The $127^{\text {th }}$ ASEE Annual Conference [Technical Session], 2020. The American Society for Engineering Education: Montreal (Virtual Conference). 
[14] L. R. Halverson and C. R. Graham, "Learner Engagement in Blended Learning Environments: A Conceptual Framework," Online Learning, 23.2, (2019): 145-178.

[15] M. H. Immordino-Yang, Emotions, learning, and the brain: Exploring the educational implications of affective neuroscience. WW Norton \& Company, 2015.

[16] T. De Fazio, A. Gilding, and G. Zorzenon, "Student learning support in an online learning environment," in ASCILITE, 2000. 